\title{
Das Wahlsystem zwischen normativer Begründung, empirischer Evidenz und politischen Interessen. Ein Kommentar zu Gerd Strohmeier sowie Franz Urban Pappi und Michael Herrmann
}

\author{
Joachim Behnke und Florian Grotz
}

In der letzten Ausgabe der ZParl diskutiert Gerd Strohmeier mehrere Beiträge zur anstehenden Reform des Bundestagswahlsystems von Behnke, Pappi / Herrmann und Lübbert, die in Heft 2/2010 der ZParl erschienen sind. ${ }^{1}$ Dabei plädiert er letztlich für ein Wahlsystem, das auf die Verbindung der Landeslisten verzichtet, und schließt sich so in der Sache dem Vorschlag von Pappi und Herrmann ${ }^{2}$ an. Wir teilen Strohmeiers Ansicht, dass eine Grundsatzdebatte über die Einführung eines mehrheitsbildenden Wahlsystems nicht (mehr) sinnvoll ist, sondern es darum geht, die Struktur des bestehenden Systems weitestgehend zu erhalten und zugleich den Vorgaben des Bundesverfassungsgerichts Rechnung zu tragen. Wir stimmen mit ihm auch darin überein, dass es keine optimale Lösung für dieses Problem gibt, da jeder verfassungsrechtlich effektive „Eingriff“ in das bestehende Wahlsystem mit (unerwünschten) „Nebenwirkungen“ verbunden ist. ${ }^{3}$ Allerdings bestreiten wir, dass das von Pappi und Hermann diskutierte Modell - das der von Union und FDP gegenwärtig favorisierten Lösung weitgehend entspricht ${ }^{4}$ - das „geeignetste“ ist. Vielmehr raten wir von seiner Einführung ab. Diese Schlussfolgerung ergibt sich aus normativ-konzeptionellen Problemen, die die Argumentation von Pappi und Herrmann aufwirft (1), aber auch aus offensichtlichen Schwierigkeiten, die sich mit den erkennbaren Auswirkungen dieses Wahlsystems (2) sowie mit dessen politischer Umsetzung (3) verbinden.

1 Vgl. Gerd Strohmeier, Die Geister, die Karlsruhe rief - eine Replik auf die Beiträge zur Wahlsystemreform in Heft 2/2010 der ZParl, in: ZParl, 42 Jg. (2011), H. 1, S. 186 - 193.

2 Vgl. Franz Urban Pappi / Michael Herrmann, Überhangmandate ohne negatives Stimmgewicht: Machbarkeit, Wirkungen, Beurteilung, in: ZParl, 41 Jg. (2010), H. 2, S. 260 - 278.

3 Ausführlich hierzu Florian Grotz, Abschied von der personalisierten Verhältniswahl? Perspektiven einer Reform des Bundestagswahlsystems, in: Eckhard Jesse / Roland Sturm (Koord.), Bilanz der Bundestagswahl 2009. Voraussetzungen, Ergebnisse, Folgen, München 2010, S. 411 - 432, S. $425 \mathrm{ff}$.

4 Vgl. Stephan Löwenstein, Nur noch wenig Zeit für die Mathematiker der Macht, in: FAZ vom 9. April 2011, S. 4. Dieser Entwurf der gegenwärtigen Regierungsparteien weicht allerdings in einem zentralen Punkt von einer bloßen Trennung der Landeslisten ab. So sollen die Sitzkontingente der Länder, die dann auf die Landeslisten der verschiedenen Parteien verteilt würden, entsprechend der Wahlbeteiligung bestimmt werden. Damit aber würde ein Effekt des negativen Stimmgewichts erhalten bleiben. Nach diesem Modell hätten zum Beispiel bei der letzten Wahl circa 12.600 Stimmen weniger für Die Linke nicht nur im Zuge des Proporzverfahrens einen Sitz weniger für die CSU bedeutet (der allerdings durch Überhangmandate „aufgefangen“ worden wäre), sondern auch einen Sitz mehr für Die Linke in Nordrhein-Westfalen, da in der nun länderbezogenen Oberverteilung ein Sitz von Bayern nach Nordrhein-Westfalen „gewandert“ wäre (eigene Berechnung). 
(1) Im Zentrum der gegenwärtigen Wahlsystemdebatte steht nicht nur das vom Bundesverfassungsgericht monierte "negative Stimmgewicht“, sondern auch und gerade das massive Auftreten von Überhangmandaten. Diesbezüglich nehmen Pappi und Herrmann eine normative Position ein, die in klarem Gegensatz zur verbreiteten Interpretation des Bundeswahlgesetzes steht. Dass die von ihnen unterstützte Trennung der Landeslisten ${ }^{5}$ die Überhangmandate bestehen ließe, bewerten sie nicht als „hinnehmbares Übel“, sondern im Gegenteil als begrüßenswerte Eigenschaft des Systems. Demnach sind Überhangmandate als „Mehrheitsbonus zugunsten des Parteilagers [...], das sich besser koordinieren kann"6 zu verstehen. Die Koordinationsfähigkeit beruht dabei auf vorab bekundeten Koalitionsabsichten der Parteien eines bestimmten Lagers. Der potentiell mehrheitsförderliche Effekt von Überhangmandaten wird damit vom Zufallsprodukt zum „wesentlichen Strukturmerkmal"7 des Wahlsystems. Der Tatsache, dass diese Charakterisierung weder den ursprünglichen Intentionen der Wahlgesetzgeber noch der über 50-jährigen einschlägigen Rechtsprechung ${ }^{8}$ entspricht, sind sich Pappi und Herrmann wohl bewusst. Die herkömmliche Interpretation sei der „fehlgeleiteten Sichtweise“ geschuldet, dass das deutsche Wahlsystem ein Verhältniswahlsystem sei. In Wahrheit habe es sich schon immer um ein „Mischwahlsystem “ gehandelt, bei dem für die beiden Mandatsklassen, die Wahlkreismandate und die Listenmandate, unabhängige Legitimationsketten bestünden: „Jedes der zwei Segmente des Parlaments ist vom Wähler legitimiert, wenn auch auf unterschiedliche Weise, und für jedes Segment steht dem Wähler eine Stimme zur Verfügung, die Erststimme für den Wahlkreiskandidaten und die Zweitstimme für die Landesliste einer Partei. "9 Der so konstruierte „Mischwahlcharakter der Legitimationskette“ scheint daher dem eines Grabenwahlsystems zu entsprechen, was durch die Betonung der "Segmente" des Parlaments noch hervorgehoben wird. Damit ignorieren Pappi und Herrmann allerdings die grundlegende Bedeutung des Verhältnisausgleichs, wie er in $\$ 6$ Abs. 2 und Abs. 4 des Bundeswahlgesetzes festgelegt ist. Demnach bezieht sich das Mischelement auf die Mechanismen der Personenauswahl, keineswegs auf den Gesichtspunkt der Repräsentation im Hinblick auf die Gesamtverteilung. Daher wird das deutsche Wahlsystem nicht nur vom rechts- und politikwissenschaftlichen Mainstream ${ }^{10}$, sondern auch vom Bundesverfassungsgericht in allen einschlägigen Urteilen durchgehend als Verhältniswahlsystem ${ }^{11}$ charakterisiert - eine Position, der sich im Übrigen auch Strohmeier anschließt. ${ }^{12}$ In der bisherigen Verfassungsrechtsprechung drückt sich der Charakter der Verhältniswahl als Gebot der Erfolgswertgleichheit

5 Es geht hierbei allerdings nicht einfach um das Verbot von Listenverbindungen, so dass dann etwa alle Landeslisten der Parteien im Zuge eines einzigen Verteilungsschritts an der proportionalen Sitzzuteilung teilnehmen würden. Das entscheidende Merkmal dieses und verwandter Lösungsvorschläge besteht darin, dass das zweistufige Verfahren beibehalten, die Reihenfolge Parteien und Länder aber gewissermaßen umgedreht wird. Zuerst werden die Sitze proportional auf die Länder verteilt, anschließend innerhalb der Länder auf die Landeslisten der Parteien.

6 Franz Urban Pappi / Michael Herrmann, a.a.O. (Fn. 2), S. 272.

7 Ebenda, S. 277.

8 Vgl. hierzu Hans Meyer, Die Zukunft des Bundestagswahlrechts, Baden-Baden 2010, S. 33.

9 Franz Urban Pappi / Michael Herrmann, a.a.O. (Fn. 2), S. 271

10 Vgl. exemplarisch Hans Meyer, a.a.O. (Fn. 8); Dieter Nohlen, Wahlrecht und Parteiensystem, Opladen 2009.

11 Häufig spricht das Bundesverfassungsgericht etwas differenzierter vom „Grundcharakter der Bundestagswahl als einer Verhältniswahl“. Vgl. stellvertretend BVerfGE 95, S. 335 - 407, S. 365.

12 Vgl. Gerd Strohmeier, a.a.O. (Fn. 1), S. 188. 
aus. Überhangmandate werden dabei als Einschränkung beziehungsweise Verzerrung dieser Erfolgswertgleichheit akzeptiert, die als Preis zur Gewährleistung anderer Elemente des Wahlsystems, insbesondere der Personenwahl, unumgänglich zu entrichten sind. Es gibt nach der Rechtsprechung des Bundesverfassungsgerichts also keine Rechtfertigung der Überhangmandate als Überhangmandate, sondern lediglich eine indirekt abgeleitete, die sich auf ihre Legitimation als Direktmandate stützt.

So wenig plausibel die Uminterpretation des bestehenden Wahlsystems durch Pappi und Herrmann ist, so sehr drängt sich die Frage auf, welche Funktion diese Argumentation für die Begründung ihres Modells hat. Offensichtlich geht es den beiden Autoren darum, die Überhangmandate positiv, also als gewollten und durch das gegebene Design intendierten Effekt, zu rechtfertigen. ${ }^{13}$ Wenn Überhangmandate kein „unschöner Nebeneffekt ${ }^{\text {“14 }}$ der personalisierten Verhältniswahl, sondern Strukturmerkmal eines „Mischsystems“ sind, dann - so die mögliche Überlegung - wäre ihr Auftreten positiv begründet, und das PappiHerrmann-Modell würde lediglich „das deutsche Wahlsystem in seiner bisherigen Form [...] bewahren"15. Wenn man aber der - aus unserer Sicht mehr als zweifelhaften - Charakterisierung als Mischwahlsystem nicht folgen möchte, bedürfte es einer eigenständigen normativen Begründung, die in der Lage wäre, die Überhangmandate als Überhangmandate zu legitimieren. An diesem Punkt eröffnen Pappi und Herrmann zwar einen weiteren Argumentationsstrang, bleiben aber die entscheidenden Antworten schuldig. Die Beibehaltung der Überhangmandate wird mit dem durch sie bewirkten „Mehrheitsbonus“ zu rechtfertigen versucht, es wird aber nicht erklärt, warum ein solcher überhaupt als wünschenswert betrachtet werden sollte. Hier verweisen die Autoren lediglich auf die vermeintliche „Erleichterung der Regierungsbildung " in Griechenland und Italien ${ }^{16}$ - Fälle, bei denen wir normalerweise nicht geneigt sind, sie als paradigmatische Beispiele für politische Stabilität anzusehen. Zudem muss - wie Pappi und Herrmann selbst einräumen - ein solcher mehrheitsbildender Effekt nicht bei jeder Wahl eintreten. Nicht weniger wahrscheinlich ist, dass „Boni“ in Form von Überhangmandaten andere Mehrheiten zerstören, die zuvor möglich waren. ${ }^{17}$ Warum aber die durch Überhangmandate entstandenen Koalitionsoptionen anderen Mehrheitskonstellationen normativ vorzuziehen sein sollten, ist in keiner Weise ersichtlich.

Aber auch die „empirischen Prämissen“ dieser Argumentation sind alles andere als eindeutig, denn Mehrheitsboni können entsprechend der Logik von Pappi und Herrmann eigentlich nur dann als erwünscht angesehen werden, wenn sie Ergebnisse einer strategischen

13 Pappi und Herrmann sind nicht die ersten, die eine solche positive Begründung der Überhangmandate versuchen. Vgl. beispielsweise Thomas Poschmann, Wahlrechtsgleichheit und Zweistimmensystem, in: Bayerische Verwaltungsblätter, 41. Jg. (1995), S. 299 - 302, Hans-Jürgen Papier, Überhangmandate und Verfassungsrecht, in: JuristenZeitung, 51. Jg. (1996), S. 265 - 274. Zu einer kritischen Diskussion des Prämienarguments vgl. Joachim Behnke, Überhangmandate: Ein (behebbarer) Makel im institutionellen Design des Wahlsystems, in: ZPol, 13. Jg. (2003), H. 3, S. 1235 - 1269. Tatsächlich nehmen Überhangmandate deutlich zu, je weniger erfolgreich die großen Parteien werden, was die Bundestagswahl 2009 demonstriert hat. Anstatt von einer Siegerprämie müsste man daher bei Überhangmandaten eigentlich von einer „Verliererprämie“ sprechen.

14 Franz Urban Pappi / Michael Herrmann, a.a.O. (Fn. 2), S. 266.

15 Ebenda, S. 278.

16 Vgl. ebenda, S. $271 \mathrm{f}$.

17 Vgl. Hans Meyer, a.a.O. (Fn. 8), S. 33; Joachim Behnke, a.a.O. (Fn. 13). 
Wählerkoordination sind, die durch explizit im Wahlkampf bekundete Koalitionsabsichten ermöglicht wird. In diesem Fall belohnen die Überhangmandate solche Bündnisse, die auch arbeitsfähig wären beziehungsweise deren Zustandekommen im Falle eines entsprechenden Wahlergebnisses gesichert wäre, da die Parteien vor der Wahl die Absicht zu ihrer Bildung erklärt haben. Stimmenkoordination wird durch die Koalitionsbekundung aber nur dann ermöglicht, wenn das sich anbietende Koordinationsmuster gleichzeitig durch die vorhandene Struktur des Parteiensystems offensichtlich wird. Bei einer bekundeten Präkoalition zwischen Union und FDP ergibt sich als ,stillschweigende“ Koordinationsmöglichkeit, mit der Erststimme die "voreingestellte“ große Partei, also die CDU, und mit der Zweitstimme die kleine Partei, also die FDP, zu wählen. ${ }^{18}$ Andere Präkoalitionen wie derzeit Rot-Grün beziehungsweise Grün-Rot, in denen der Größenunterschied zwischen beiden Parteien nicht so deutlich ist, würden keineswegs ebenso eindeutige Signale aussenden, was ihr Potenzial zur Koordination beeinträchtigen würde. Für die Anhänger einer Präkoalition aus einer 40 Prozent- und einer 5 Prozent-Partei erweist es sich als offensichtlich, dass die Erststimmen auf die große Partei konzentriert werden sollten, da sie nur dort zur Gewinnung von Direktmandaten beitragen können. ${ }^{19}$ Sie verfügen mit der „voreingestellten“ großen Partei über eine Art von Schelling'schem „focal point“ zur Lösung ihres Koordinationsproblems. Eine Präkoalition zwischen zwei Parteien, die beide auf ein Potenzial von 22 bis 24 Prozent geschätzt werden, hat diesen Punkt hingegen nicht, da es für die Anhänger nicht klar erkennbar ist, auf welche der beiden Parteien sie ihre Erststimmen konzentrieren sollten. Hier könnte das Koordinationsproblem nur durch explizite Wahlabsprachen, das heißt zum Beispiel durch Verzicht auf Nominierungen, gelöst werden. Implizite oder stillschweigende Koordination bleibt aber schwierig. Die Fähigkeit bestimmter Präkoalitionen zur stillschweigenden Koordination ihrer Anhängerschaft bezüglich der Stimmabgabe hängt also nicht von der Stärke des bekundeten Willens zur Koalitionsbildung ab. Es kann sogar sein, dass der Wille zur Bildung einer Koalition aus zwei mittelgroßen Parteien deutlicher ausgedrückt wird als der zur gegnerischen Koalition aus einer großen und einer kleinen Partei. Dennoch würde es letztere sein, die von den Überhangmandaten profitiert. Dies entspricht jedoch offensichtlich nicht der normativen Stoßrichtung von Pappi und Herrmann. Außerdem ist nur der kleinere Teil der Überhangmandate auf strategisches Wählen, also Stimmensplitting, zurückzuführen. Überhangmandate entfallen daher nicht unbedingt auf das besser koordinierte Lager, sondern kommen einfach zu Gunsten der größten Partei zustande, solange diese immer noch klar vor den anderen Parteien und gleichzeitig deutlich unter 50 Prozent der Zweitstimmen liegt. Für einen Mehrheitsbonus, der nur für die größte Partei anfällt, kann aber das Rechtfertigungsargument von Pappi und Herrmann offensichtlich nicht in Anspruch genommen werden.

18 Im spieltheoretischen Sinne würden die „voreingestellten“ Größen der Parteien als Signale für diese Form stillschweigender Koordination gedeutet werden; das damit sich herauskristallisierende Splittingmuster wäre dann eines, das sowohl von CDU- als auch FDP-Anhängern umgesetzt würde. Geht man hingegen lediglich von Anhängern einer kleinen Partei aus, die ihre ansonsten „wertlose“ Erststimme dem Kandidaten einer nahestehenden Partei geben, so wäre diese Verhalten zwar unter Umständen nutzenmaximierend und in diesem Sinne rational, es gäbe aber in diesem Zusammenhang keine explizite Koordinationsproblematik.

19 Die spieltheoretisch reine optimale Lösung würde allerdings eventuell sogar genau umgekehrt aussehen. 
(2) Obwohl Pappi / Herrmann und Strohmeier dasselbe Reformmodell favorisieren, schätzen sie seine empirischen Auswirkungen unterschiedlich ein. Während Strohmeier die Anzahl der Überhangmandate im neuen System eher auf geringerem Niveau anzusetzen scheint als bisher ${ }^{20}$, messen Pappi und Herrmann der mehrheitsbildenden Wirkung der Überhangmandate beträchtliche Bedeutung zu: Zwar sei ein Mehrheitsbonus „keineswegs garantiert“, doch könnte man „auch mit diesem Mischsystem alternierende Regierungsmehrheiten zustande bringen “21. In der Tat lassen sich dessen genaue Effekte unter einem dynamischen Fünfparteiensystem nur schwer prognostizieren. Im Unterschied zu den anderen Reformvorschlägen in ZParl 2/2010 sind jedoch in diesem System durchaus (partei-) politische Konstellationen denkbar, unter denen deutlich mehr Überhangmandate entstehen könnten als schon 2009. ${ }^{22}$

Dies wäre dann nicht nur politisch brisant, zumal wenn dadurch die Mehrheitsverhältnisse im Bundestag beeinflusst würden, sondern hätte auch verfassungsrechtlich prekäre Konsequenzen: Entgegen einer gelegentlich geäußerten Auffassung ist die einschlägige Rechtsprechung keineswegs so zu interpretieren, dass Überhangmandate verfassungsrechtlich unbedenklich sind. In dem bisher ausführlichsten Urteil des Bundesverfassungsgerichts vom 10. April $1997 \mathrm{kam}$ es zu einem Patt von 4:4 Stimmen, womit zwar der Status quo erhalten blieb. Aber selbst jene Richter, die seinerzeit Überhangmandate nicht per se als verfassungswidrig erachteten, sahen doch Grenzen der Zumutbarkeit. Insbesondere interpretierten sie die Fünfprozenthürde auch als „Anhalt [...], nach dem der Abweichung [...] der proportionalen Verteilung der Sitze nach dem Ergebnis der für die Parteien abgegebenen (Zweit)Stimmen eine Grenze gesetzt ist "23. Im selben Urteil äußerten sich die Richter noch grundsätzlicher: „Der Grundcharakter der Wahl als Verhältniswahl lässt eine Differenzierung des Gewichts der für die Parteien abgegebenen Stimmen nicht unbeschränkt zu [...] Werden diese Grenzen überschritten, weil sich Verhältnisse einstellen, unter denen Überhangmandate von Wahl zu Wahl regelmäßig in größerer Zahl anfallen, entfernt sich das Wahlverfahren von den Grundentscheidungen des Gesetzes. Aus diesen Anforderungen an das Wahlverfahren kann sich einerseits eine Schranke für den Gestaltungsspielraum des Gesetzgebers wie andererseits aufgrund der Änderung der tatsächlichen Verhältnisse auch ein Handlungsauftrag ergeben." 24 Die 16 Überhangmandate von 1994, die der Auslöser für die Verfassungsklage gewesen waren, wurden vom Gericht offensichtlich als eine solche „größere Zahl“ empfunden. Da in drei der vier darauf folgenden Bundestagswahlen Überhangmandate in dem gleichen oder noch größeren Umfang entstanden sind, könnte man demnach von einer gewissen „Regelmäßigkeit“ des Auftretens sprechen und mithin einen

20 Vgl. Gerd Strohmeier, a.a.O. (Fn. 1), S. 192. Inwiefern sich eine Trennung der Landeslisten auf die Entstehung von Überhangmandaten auswirkt, hängt auch davon ab, wie man die Ländersitzkontingente von der Wahlbeteiligung beziehungsweise den Stimmenanteilen jener Parteien, die an der Proporzverteilung teilnehmen, abhängig macht. Bei starren Sitzkontingenten der Länder (wie bei Pappi und Herrmann) würden in Ländern mit unterdurchschnittlicher Wahlbeteiligung im Vergleich zum aktuellen Verfahren zwar eher weniger Überhangmandate anfallen, in Ländern mit überdurchschnittlicher Wahlbeteiligung jedoch eher mehr.

21 Franz Urban Pappi / Michael Herrmann, a.a.O. (Fn. 2), S. 272 ff.

22 Vgl. Joachim Behnke, Überhangmandate und negatives Stimmgewicht: Zweimannwahlkreise und andere Lösungsvorschläge, in: ZParl, 41 Jg. (2010), H. 2, S. 247 - 263, S. 250.

23 BVerfGE 95, S. 335 - 407, S. 366.

24 Ebenda, S. 365. 
Handlungsauftrag ableiten, dies zu korrigieren. Neben dem explizit formulierten Auftrag an den Gesetzgeber durch das Urteil zum negativen Stimmgewicht von 2008 gäbe es also auch einen konditional formulierten zur Abschaffung der Überhangmandate, wenn man sich an das Urteil von 1997 hält. Auch in empirischer Hinsicht birgt das von Pappi und Herrmann favorisierte Modell also zu viele unwägbare beziehungsweise problematische Konsequenzen, die seine Einführung als höchst fragwürdig erscheinen lassen.

(3) Selbst wenn man die benannten normativ-konzeptionellen und empirischen Schwierigkeiten ausklammern könnte, wäre es auch mit Blick auf die gegebene parteipolitische Interessenkonstellationen nicht ratsam, diese Reformoption umzusetzen. Wie eingangs angesprochen, wird der Vorschlag separater Landeslisten mit einer länderbezogenen „Oberverteilung“ und einer parteienbezogenen „Unterverteilung“ derzeit von der Berliner Regierungskoalition verfolgt, während die Oppositionsparteien - allen voran die SPD ${ }^{25}$ - dies strikt ablehnen. Die jeweiligen Gründe sind offensichtlich: Wahlsystemfragen sind immer auch Machtfragen. Während die Union - womöglich in einer zu optimistischen Extrapolation der letzten Wahlergebnisse - davon auszugehen scheint, dass die sich herauskristallisierende neue Struktur des Parteiensystems in punkto Überhangmandate zu ihren Gunsten wirken dürfte, sieht die SPD diesen Vorschlag eben deswegen kritisch. Natürlich stünde es CDU/CSU und FDP frei, das Wahlgesetz in ihrem Sinn mit einfacher Bundestagsmehrheit zu ändern. Allerdings würden sie sich dadurch nicht nur dem generellen Vorwurf der institutionellen Machtabsicherung aussetzen. Vielmehr könnte auch - angenommen, es entstünde unter dem System getrennter Landeslisten eine hinreichend große Zahl von Überhangmandaten - das Bundesverfassungsgericht aus den genannten Gründen erneut mit dem Wahlsystem befasst werden. ${ }^{26}$ Dass ein solcher machtpolitisch motivierter, juristisch ausgetragener Parteienkonflikt der Legitimität, die nach Dieter Nohlen das übergeordnete und damit wichtigste Gütekriterium für demokratische Wahlsysteme darstellt ${ }^{27}$, abträglich wäre, braucht nicht weiter erläutert zu werden.

Insgesamt folgt aus den vorgetragenen Überlegungen, dass das Modell von Pappi und Herrmann für den gegenwärtigen Reformkontext ungeeignet ist. Es ist sicherlich richtig, dass auch die anderen in der Diskussion befindlichen Optionen - wie die Schaffung von Ausgleichsmandaten, die Kompensation der Überhangmandate über Landeslisten oder die Einrichtung von Zweipersonenwahlkreisen - je spezifische Nachteile haben; in der Summe fallen diese aber deutlich weniger schwer ins Gewicht als jene des Pappi-Herrmann-Vorschlags.

$\mathrm{Zu}$ Strohmeiers Kritik an den anderen Vorschlägen ist abschließend folgendes zu bemerken: Es ist vollkommen richtig, dass sowohl die Einrichtung von Zweierwahlkreisen als auch die Absenkung des Anteils der Direktmandate auf zum Beispiel 40 Prozent das Auftreten von negativen Stimmgewichten nicht „logisch“ beseitigen würde, das heißt, der Effekt des negativen Stimmgewichts wäre theoretisch weiterhin möglich. Allerdings würden diese Vorschläge zur faktischen Vermeidung beziehungsweise drastischen Verminderung

25 Vgl. Thomas Oppermann, Nicht ohne Ausgleichsmandate, in: FAZ vom 7. Januar 2011, S. 10.

26 Dass dies kein völlig unrealistisches Szenario darstellt, zeigt unter anderem folgende Äußerung von Thomas Oppermann, dem Ersten Parlamentarischen Geschäftsführer der SPD-Bundestagsfraktion: „Das Bundesverfassungsgericht hat Überhangmandate - noch - nicht verboten. Sie werden aber mit stetig steigender Zahl verfassungsrechtlich kritisch.“ Ebenda.

27 Vgl. Dieter Nohlen, a.a.O. (Fn. 10), S. 155 ff. 
von Überhangmandaten führen, die wir - wie hier nochmals deutlich wurde - als das eigentliche Problem des jetzigen Wahlsystems betrachten. Kommen gar keine Überhangmandate mehr zustande beziehungsweise sind keine in Sichtweite, entsteht auch kein Effekt des negativen Stimmgewichts, denn dieses ist im vorliegenden Wahlsystem an Überhangmandate geknüpft. Man hätte dann das Problem des negativen Stimmgewichts „en passant“ mit abgewickelt. Sollten aber immer noch Überhangmandate vorkommen, würden sie nach aller Wahrscheinlichkeit in deutlich vermindertem Umfang auftreten. In diesem Fall könnten sie durch Ausgleichsmandate kompensiert werden, ohne dass es zu einer nicht mehr hinnehmbaren Vergrößerung des Bundestags käme. Die Zweipersonenwahlkreise beziehungsweise die Absenkung des Anteils der Direktmandate würden gewährleisten, dass die Ausgleichsmandate zahlenmäßig begrenzt bleiben. In der Regel aber wäre damit zu rechnen, dass Ausgleichsmandate gar nicht benötigt werden, da erst gar keine Überhangmandate anfallen würden. Eine Ausgleichsregelung ist daher nur als flankierende Maßnahme zu den Zweipersonenwahlkreisen oder der Absenkung der Direktmandate vonnöten. Das negative Stimmgewicht wäre beseitigt, weil entweder keine Überhangmandate mehr anfielen oder die noch vereinzelt auftretenden durch Ausgleichsmandate neutralisiert würden.

Interpretiert man das negative Stimmgewicht allerdings sehr wörtlich beziehungsweise sehr eng, dann schaffen Ausgleichsmandate dieses strenggenommen nicht ab, da durch weniger Stimmen immer noch mehr Sitze für eine Partei entstehen könnten. Die verfassungsrechtliche Problematik des negativen Stimmgewichts begründete das Bundesverfassungsgericht im Urteil vom 3. Juli 2008 allerdings mit dem Postulat der Erfolgswertgleichheit. Diese interpretierten die Richter so, dass damit verbunden sei, dass eine Stimme eine „positive Wirkung "28 für die abgegebene Partei entfalten müsse. Dies wirft nun die interessante Frage auf, was darunter aus der Sicht des Wählers zu verstehen sein könnte. Vermutlich beabsichtigt der Wähler mit der Abgabe seiner Stimme nicht unbedingt, zur Gewinnung eines weiteren Mandats für seine Partei beizutragen; vielmehr geht es ihm darum, diese zu unterstützen. Auf jeden Fall möchte er ausschließen, ihr zu schaden. Der Nutzen oder Schaden für eine Partei aber ergibt sich durch den relativen Vor- oder Nachteil, den ein weiteres beziehungsweise verloren gegangenes Mandat für eine Partei im Vergleich zu anderen bedeutet. Ausgleichsmandate würden diese Form des Vorteils beziehungsweise Schadens, wenn er durch Überhangmandate zustande kommt, neutralisieren. Insofern ist es plausibel anzunehmen, dass Ausgleichsmandate eine verfassungsrechtlich akzeptable Lösung zur Beseitigung des negativen Stimmgewichts darstellen. Auch Strohmeier ist offensichtlich dieser Ansicht, da er Ausgleichsmandate nur wegen der damit verbundenen Aufblähung des Bundestags ablehnt.

In jedem Fall täten die Bundestagsparteien gut daran, baldmöglichst eine Lösung herbeizuführen, die nicht nur normativen und funktionalen Minima genügt, sondern auch auf einem breiten politischen Konsens beruht. 